\title{
Innovar en compañía: el Repositorio de Innovación Educativa de la UNAM
}

\author{
Roberto Santos Solórzano y Patricia González-Flores
}

\begin{abstract}
Resumen
Este artículo describe el Repositorio de Innovación Educativa -una plataforma digital que da acceso a diversos recursos y productos sobre innovación educativa- como un mecanismo que busca facilitar la sistematización y visibilización de las innovaciones educativas que se realizan en la Universidad Nacional Autónoma de México (UNAM). Además, se aborda la importancia de la innovación, y los retos y dificultades para implementarla en el contexto universitario. Por último, se exponen las fortalezas de innovar en colaboración y el papel que la Coordinación de Desarrollo Educativo e Innovación Curricular (CODEIc) de la unAm asume en este fin.
\end{abstract}

Palabras clave: innovación educativa, repositorio, plataforma digital, CODEIC, UNAM.

\section{Collaborating to innovate: The Educational InNovation Repository at UNAM}

\begin{abstract}
This article describes the Educational Innovation Repository at the National Autonomous University of Mexico (UNAM), an online platform designed to provide access to literature and resources that deal with the transformation of teaching and learning practices, conceived as a strategy to visualize and organize systematically innovative initiatives at this university. It also addresses the key role that educational innovation plays for higher education institutions and the challenges to implement new ideas and processes in this context. Finally, the potential of collaboration in order to promote educational changes is analyzed, as well as the strategy that unAM'S CODEIC has designed to move forward in this direction.
\end{abstract}

Keywords: educational innovation, repository, online platform, codeIc. 


\section{Roberto Santos Solórzano}

Especialista en recuperación y manejo de información científica y académica en la Web, gestión de bases de datos bibliográficas, curación digital, normalización y estandarización. Pasante de Maestro en Docencia para la Educación Media Superior (MADEMS) y Biólogo por la Facultad de Ciencias, ambos de la unAm. Ha participado en la coordinación, diseño e impartición de cursos sobre el desarrollo de competencias básicas y el uso de herramientas de la Web 2.0 para el manejo de literatura científica en diversas instituciones públicas y privadas. Actualmente es Jefe de Departamento de Innovación Educativa en la Coordinación de Desarrollo Educativo e Innovación Curricular (CODEIC).

\section{Patricia González Flores}

patricia.gonzalez@codeic.unam.mx

Especialista en tecnología educativa y educación a distancia con más de 20 años de experiencia en implementación de proyectos educativos en instituciones públicas y privadas, diseño de cursos en línea, así como formación docente. Entre sus publicaciones especializadas, se incluye el libro Essentials of Online Teaching: A Standards-Based Guide editado por Routledge. Es licenciada en Pedagogía por la Universidad Nacional Autónoma de México (UNAm) y maestra en Medios de Comunicación por la New School for Social Research (Nueva York, Estados Unidos). Funge actualmente como Subdirectora de Innovación Educativa de la de la Coordinación de Desarrollo Educativo e Innovación Curricular (CODEIC). 


\section{Introducción}

Las universidades tienen la responsabilidad de adaptarse y responder a los desafíos de esta época, marcada por turbulencias y cambios cada vez más acelerados. En dicho escenario, la innovación educativa adquiere importancia y significado, ya que se refiere a la introducción y adopción de una idea, modelo, proceso, servicio o recurso que consideramos "nuevo" y que genera algún tipo de mejora en los procesos de enseñanza y aprendizaje (Barraza, 2005; Banerjee, 2016; Tierney \& Lanford, 2016; Zabalza \& Zabalza, 2003). Este impacto positivo la posiciona como una alternativa para resolver los retos y demandas que enfrentan las instituciones educativas. En particular, se le vincula con la mejora en la calidad de los aprendizajes, el fomento de la equidad, la eficiencia y el impulso de las transformaciones que requiere la sociedad actual (Organisation for Economic Co-operation and Development [OECD], 2014).

De esta forma, se generan altas expectativas sobre "lo nuevo" de la innovación. Sin embargo, calificar cualquier idea, modelo, producto, servicio o proceso como novedoso resulta muy complicado, pues depende del contexto y el momento en el cual se introduce: algo puede ser considerado como nuevo si no se ha utilizado antes en un espacio en particular, sin importar que en otro lado ya sea conocido. Una "misma" innovación no será necesariamente reconocida como tal por todas las organizaciones, sean estas escuelas o facultades o universidades. Por lo tanto, la definición específica de las prácticas y los procesos que son innovadores debe ser delimitada y reconocida por la comunidad misma donde éstos tienen lugar (Rogers, 2003; Tierney \& Lanford, 2016).

Aunque la necesidad de innovar es constante en el discurso educativo, si el término se usa de manera descontextualizada y excesiva, se corre el riesgo de que no se entienda qué es, se descalifique o incluso se cuestione su existencia, en el marco de ideas de sentido común como "no hay nada nuevo bajo el sol" o "eso ya se ha visto antes o en otro lado". Por otra parte, para las universidades es importante crear las condiciones necesarias para documentar innovaciones educativas que surgen en su interior, sistematizar e investigar cómo éstas se generan, si son apropiadas para su comunidad y qué impacto tienen en el logro de las funciones sustantivas de la institución. De este modo, será posible comprender cómo se transforma la institución y apropiarse de procesos de diseño y estrategias que contribuyan a impulsar los procesos de cambio (Tierney \& Lanford, 2016).

\section{Retos de la innovación educativa}

Para cualquier organización, innovar es difícil; ya que implica modificar prácticas y comportamientos, modelos mentales, cultura organizacional e ideas preconcebidas sobre cómo tener éxito en su misión (Banerjee, 2016). 
Estos cambios resultan particularmente complejos para las universidades cuya enorme tradición histórica y social se extiende a través de los años y se sustenta en ideas arraigadas entre directivos, profesores y estudiantes, en torno a cómo se enseña y se aprende. Surgen así tensiones y contradicciones entre las costumbres que caracterizan el ser de las universidades y los retos de la modernización (Casanova, 2015).

Hoy en día, muchas instituciones de educación superior se han propuesto modificar sus prácticas de manera sistemática. Para lograrlo han buscado múltiples y variadas estrategias, tales como el establecimiento de áreas o unidades dedicadas a promover la innovación, la creación de programas de estímulos y premios que apoyen las iniciativas novedosas de sus comunidades y que reconozcan las aportaciones innovadoras de sus académicos.

Sin embargo, en la práctica cotidiana es común que los docentes que buscan innovar enfrenten contradicciones que obstaculizan el proceso de cambio. Por ejemplo, pueden haber diseñado e implementado nuevas formas de enseñar, distintas a la cátedra tradicional, que requieren una interacción horizontal entre docentes y estudiantes. Pero, en tal caso, el obstáculo es que tienen que trabajar en espacios de aulas que son similares a las de hace un siglo o más, que favorecen relaciones verticales y unidireccionales.

\section{Innovación educativa en la UNAM}

Al respecto, la UNAM no es excepción en este universo de instituciones de educación superior, ni en el interés en innovar, ni en los retos que debe enfrentar para lograrlo. Para ello, desde hace tres décadas, cuenta con un programa institucional orientado a promover la innovación y el mejoramiento de la enseñanza en el bachillerato y la licenciatura, hoy conocido como el Programa de Apoyo a Proyectos para Innovar y Mejorar la Educación (PAPIME). Durante sus 29 años de operación, entre 1989 y 2018, el PAPIME ha otorgado recursos a académicos, que han realizado casi 9000 proyectos. Además, en 2015 se creó la Coordinación de Desarrollo Educativo e Innovación Curricular (CODEIC) como una dependencia universitaria con la función entre muchas otras, de promover la innovación educativa.

En la UNAM, entre su numerosa planta académica que alcanza 41,318 docentes (UNAM, 2019), hay muchos profesores e investigadores innovadores que día a día ponen en práctica nuevas formas de implementar los planes y programas de estudio, experimentan diversas estrategias de enseñanza, crean recursos didácticos que permiten a los estudiantes acercarse a los contenidos de maneras no tradicionales e introducen herramientas digitales de vanguardia en su docencia. Algunos incluso retoman y adaptan innovaciones diseñadas en otras instituciones. 
Figura 1. Difusión de las innovaciones (a partir de Rogers,
De acuerdo con la investigación de Rogers (2003), acerca de la difusión de las innovaciones, en una población sólo $2.5 \%$ son sujetos innovadores. Ellos desarrollan las nuevas ideas que serán utilizadas por los adoptadores tempranos (13.5\%). Así, las innovaciones se difunden hacia los otros miembros de la comunidad, primero a una mayoría temprana que corresponde a 34.5\% de la población y luego a la mayoría tardía que asciende a otro 34\% (ver figura 1). En el caso de la población de la unAm, 2.5\% de los innovadores equivaldría a 1,033 académicos que intentan transformar sus prácticas de enseñanza.

\section{MODELO DE DIFUSIÓN DE LAS INNOVACIONES DE ROGERS}

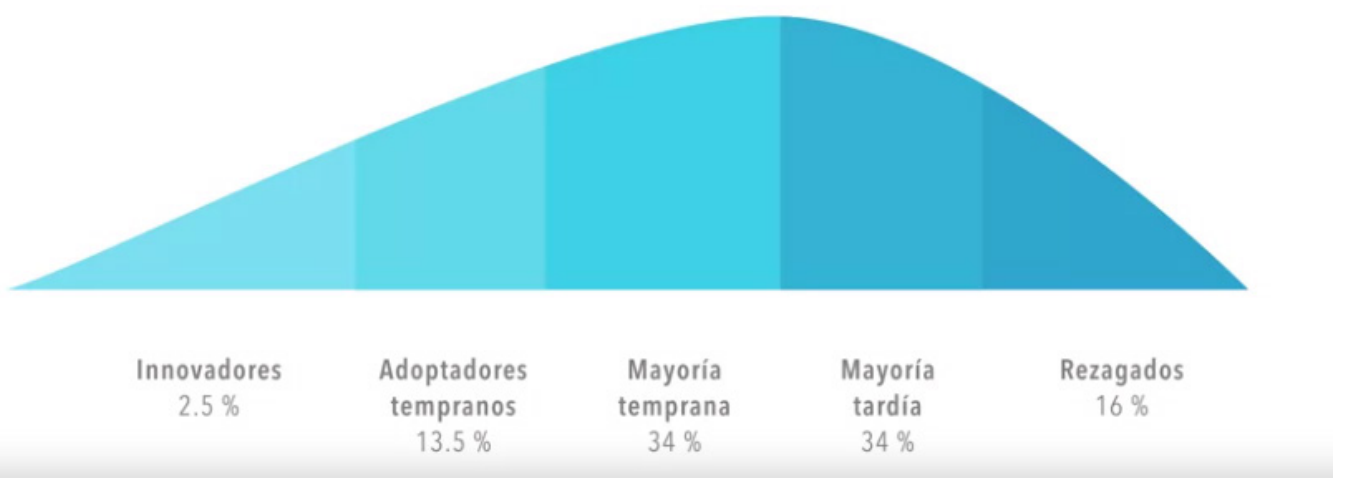

Muchas de las innovaciones educativas que se generan en la unAm son presentadas en congresos y seminarios, o compartidas con los cuerpos académicos y los grupos de pares. Otros profesores han optado por construir sus propias páginas web o alojar sus materiales en diferentes medios, pues a pesar de la existencia de muchos portales, páginas, recursos y aplicaciones institucionales, ninguno de ellos está dedicado a la innovación educativa. En consecuencia, la información sobre innovación educativa está dispersa en distintas fuentes y es poco estandarizada, lo que dificulta que los docentes o cualquier persona interesada en innovar puedan recuperarla y analizarla.

En la CODEIc, se identifica que uno de los retos en la unAm reside en integrar, sistematizar y visibilizar la innovación educativa que ya realizan los académicos con la finalidad de analizarla, difundirla y diseñar intervenciones puntuales para potenciar su impacto, una tarea sumamente compleja por múltiples causas estructurales y organizacionales, entre las que se encuentran:

- El tamaño de la institución. Además de los más de 40,000 académicos arriba mencionados, existe un cuerpo estudiantil de 356,530 y numerosas entidades (15 facultades, 8 escuelas, 34 institutos, 14 planteles de bachillerato, centros y otras sedes educativas) (UNAM, 2019). 
- La amplia distribución a nivel nacional, en las 32 entidades federativas y su presencia a nivel internacional en 9 países (UnAM, 2019).

- La variedad de planes y programas de estudios. Hay tres planes de bachillerato, 129 licenciaturas, 41 programas de posgrado y 43 especializaciones (UNAm, 2019; Hernández, 2020); y cuatro áreas del conocimiento (físico matemáticas y de las ingenierías; biológicas, químicas y de la salud; ciencias sociales; humanidades y artes).

- La enorme tradición histórica-social que se extiende por más de cinco siglos y que muchas veces dificulta el cambio de comportamientos, modelos mentales y teorías.

- La dificultad de establecer un consenso sobre qué se entiende por innovación educativa en una institución tan grande y diversa.

\section{Un repositorio para visibilizar y estudiar la innovación educativa de la UNAM}

La sistematización y visibilización de innovaciones educativas no es un tema menor ni fácil de atender. Una de las soluciones que están adoptando las universidades en las últimas décadas consiste en el desarrollo de repositorios, entendidos como espacios digitales que rompen las barreras geográficas y temporales de la comunidad educativa, constituyen entornos inspiradores, reflejan la cultura y generan sinergias que propicien la mejora de la enseñanza y el aprendizaje (Lagzian, Abrizah \& Wee, 2015).

1 Se remonta a la creación en 1553 de la Real y Pontificia Universidad de México, en la Nueva España.

\footnotetext{
2 Puede ser que los recursos se originaron directamente en medios digitales o que fueron digitalizados con el fin de preservarlos y difundirlos.
}

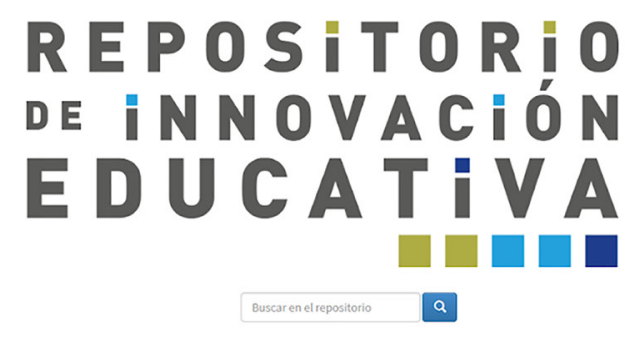

Un espacio para compartir innovaciones en educación

Un repositorio es un sistema de información que recopila, almacena, difunde y preserva los recursos generados por la población de una institución en formato digital ${ }^{2}$ Se trata de un acervo digital que se pensó para compilar la investigación original pero que, a través de los años, se ha adaptado para aceptar 
${ }^{3}$ Cátedra de Investigación de Innovación en Tecnología y Educación (REA), del Instituto Tecnológico y de Estudios Superiores de Monterrey (http:// www.ruv.itesm.mx/convenio/ catedra/).

\footnotetext{
${ }^{4}$ Por ejemplo, el Observatorio de Innovación Educativa (https:// observatorio.tec.mx/), del Tec de Monterrey, monitorea, difunde e impulsa la innovación educativa. El Repositorio de Buenas Prácticas de Innovación Educativa (http://www.buenaspracticas.net/) del Ministerio de Educación, Cultura y Deporte de España difunde trabajos sobre innovación educativa. Sin embargo, en ambos casos, no se cumplen con todas las características de estandarización e interoperabilidad que tiene un repositorio y que son importantes para organizar, buscar, sistematizar y transferir la información sobre innovación educativa.
}

otros tipos de materiales propios de la institución. De esta manera, ofrece a la comunidad un lugar para consultar, en acceso abierto (open access), imágenes, videos, información sobre proyectos, entre muchos otros recursos. También puede funcionar como una herramienta para una distribución más amplia de los productos, pues permite transferir y recibir información de otros repositorios para utilizarla de diferentes formas; a esta característica se le conoce como interoperabilidad (Lagzian, Abrizah, \& Wee, 2015).

Debido a su utilidad, el número de repositorios en todo el mundo ha crecido rápidamente; en menos de dos años se crearon 1,621 nuevos repositorios, de manera que para septiembre de 2018 ya existían 3,691, y para febrero de 2020 ya son 5,312 (Jisc, s.f.). Ni México ni la unam han estado ajenos a esta tendencia. En 2012, se inició el proyecto de la Red Mexicana de Repositorios Institucionales (REMERI), con el fin de crear una red de repositorios digitales de instituciones de educación superior (REMERI, 2012-2020). En 2016, se puso en marcha la iniciativa del Repositorio Nacional como parte de la Estrategia Nacional para Democratizar la Información Científica, Tecnológica y de Innovación del Consejo Nacional de Ciencia y Tecnología (CONACYT, 2020).

En 2009, la unAm estableció la Red de Acervos Digitales (RAD-UNAm), una red de repositorios universitarios digitales para apoyar a las dependencias y entidades en el manejo y diseminación de los productos digitales de su actividad académica. Para 2019, la Coordinación de Colecciones Universitarias Digitales (CCUD) se transformó en la Dirección General de Repositorios Universitarios (DGRU), con el objetivo de dotar a todos los repositorios digitales universitarios de normatividad metodológica, técnica y jurídica para así integrar un repositorio institucional (RI-UNAM).

Curiosamente, a pesar de los miles de repositorios existentes, los cientos de universidades que se encargan de ellos y las numerosas iniciativas que promueven su desarrollo, cuando buscamos un repositorio de innovación educativa en acceso abierto, los resultados fueron muy escasos. Por ejemplo, Opendoar, uno de los más grandes e importantes directorios internacionales de repositorios, no tiene indizado ninguno sobre este tema. Sucede lo mismo con el Repositorio Nacional, mientras que la Remeri sólo cuenta con un registro. ${ }^{3} \mathrm{La}$ ausencia de repositorios de innovación educativa no quiere decir que no existan otro tipo de acervos digitales, portales y sitios web en esta materia. ${ }^{4}$

Para atender esta necesidad, la codeıc desarrolló el Repositorio de Innovación Educativa, una plataforma digital para almacenar, organizar, preservar, difundir y proporcionar acceso abierto a los recursos sobre innovación educativa, producto de la docencia y la investigación en la unAm. Su objetivo es facilitar que los docentes compartan con la comunidad académica universitaria materiales bibliográficos, audiovisuales, didácticos o cualquier otro tipo de recurso sobre la transformación de la enseñanza y el aprendizaje en educación superior y media superior. Este repositorio está pensado como un espacio virtual de consulta que 
divulgue y propicie el intercambio de mejores prácticas, resguarde creaciones poco convencionales de académicos universitarios, así como investigación sobre la formación universitaria. Con la finalidad de garantizar su integración tanto al RI-UNAM como a las redes internacionales de repositorios, el Repositorio de Innovación Educativa está basado en los Lineamientos Generales sobre Acceso Abierto de la unAm y en estándares internacionales. Además, todos sus registros fueron revisados con el propósito de garantizar su pertinencia y fiabilidad.

En su desarrollo, el Repositorio de Innovación Educativa fue alimentado gracias a la recuperación de información de distintas colecciones en la web, por los productos generados en la propia CODEIC y la transferencia de información sobre proyectos e información del ya citado papIME. Todos estos contenidos están organizados en cuatro comunidades o grupos de recursos: Literatura de innovación educativa, Investigación en Educación, Mediateca y Proyectos. Las dos primeras almacenan publicaciones científicas sobre innovación educativa e investigación en educación, respectivamente; la tercera contiene experiencias y archivos de los eventos y concursos académicos organizados por la Subdirección de Innovación Educativa de la CODEIc; y la cuarta incluye información sobre 749 proyectos realizados por universitarios con el apoyo del PAPIME entre 2012 y 2016.

A partir del lanzamiento del Repositorio de Innovación Educativa, en marzo de 2020, será importante integrar otras innovaciones generadas por la comunidad docente, ya sean aquellas realizadas con otras fuentes de financiamiento institucional o -quizá aún más importante- las iniciativas elaboradas por los propios académicos. El Repositorio estará disponible para que todos los profesores e investigadores de la UNAM puedan difundir sus esfuerzos por transformar la enseñanza y el aprendizaje de sus estudiantes. Aquellos interesados en implementar algún tipo de innovación educativa podrán tomar como punto de partida las experiencias y productos generados por otros universitarios.

\section{Visibilizar para innovar de manera colaborativa}

Promover la innovación educativa representa una enorme exigencia para cualquier institución, pues demanda generar relaciones entre la visión organizacional, las distintas entidades y los individuos que la conforman; así como entre funcionarios, investigadores, docentes y estudiantes, cada uno con pensamientos y formas diversas de entender el mundo. Se requiere fomentar un ambiente y una cultura de innovación, donde se facilite el desarrollo e implementación de ideas transformadoras y se promuevan las conexiones entre los innovadores y los adoptadores tempranos, así como entre éstos últimos y la mayoría temprana. Se generará entonces una cadena de valor positiva y constructiva que contribuya a la difusión y adopción de las innovaciones educativas. Un ambiente de innovación será más fuerte mientras más nexos profundos tenga e invite a la colaboración y la coinnovación (Body \& Habbal, 2016). 


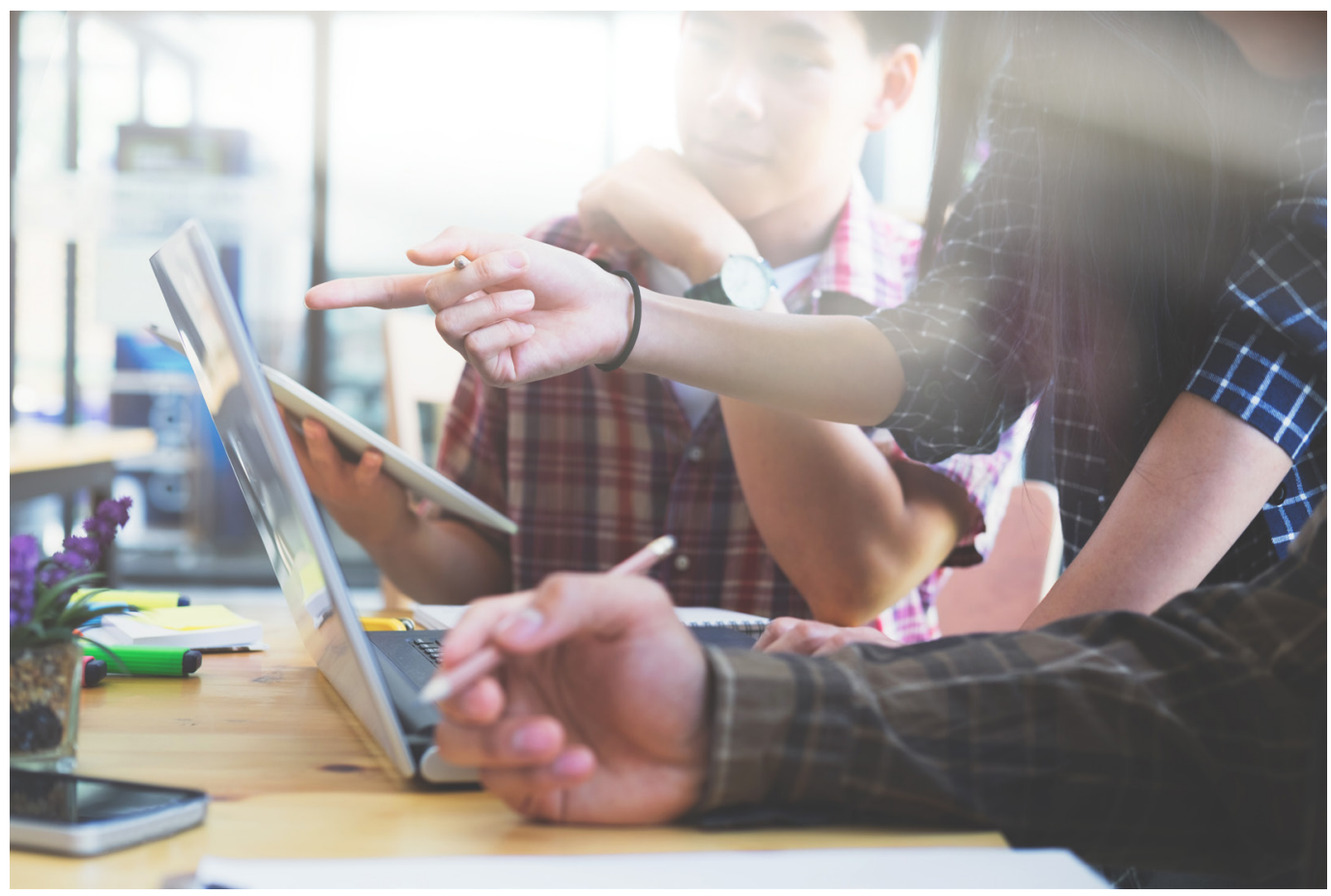

Innovar en solitario puede ser una ardua tarea. Al implementar "lo nuevo" en nuestra docencia, nos posicionamos en un terreno desconocido, en los límites de nuestro conocimiento y experiencia. Innovar requiere salir de nuestra zona de confort, a pesar de que quizá los resultados no sean ideales. La innovación demanda identificar, enfrentar y cuestionar nuestros prejuicios; también implica asumir el riesgo de que nuestras innovaciones no funcionen como las planeamos. Todo ello, en condiciones normalmente no ideales y retadoras del trabajo docente: grupos numerosos de alumnos, programas de estudio extensos, tiempo de clase corto y carencia de infraestructura.

La CODEIC busca impulsar la innovación educativa en la UNAM como un proceso colectivo que se facilita cuando profesores y estudiantes de diferentes disciplinas, competencias, formas de pensar y con distintos motivos interactúan para crear nuevas soluciones a problemas comunes. Un paso en esta dirección es el Repositorio de Innovación Educativa como un espacio dinámico de consulta, participación y discusión que constituya un "motor de la innovación".

En este sentido, como muestra, ¿sabías que en la unam hay múltiples iniciativas que exploran el uso de recursos y entornos tridimensionales conocidos como 3D? ¿Que se busca utilizarlos para favorecer aprendizajes tan disímiles como la experimentación en ciencias básicas en la Escuela Nacional Preparatoria, la odontología tanto en el campus cu como en la fEs Zaragoza, el dibujo en la Facultad de Artes y Diseño, el análisis de estructuras de construcción en la Facultad de Arquitectura, la atención a la salud mental en la Facultad de Psicología o el estudio de la diversidad animal en la fEs Iztacala? Además, el 
nivel de complejidad de estas innovaciones educativas es muy variado y abarca desde el uso de objetos digitales tridimensionales, hasta el diseño de entornos inmersivos. ${ }^{5}$

La CODEIC obtuvo esta información realizando una búsqueda en el Repositorio de Innovación Educativa para identificar los proyectos PAPIME realizados en 5 años, entre 2012 y 2016 sobre este tema. Es probable que a éstos se sumen otras innovaciones educativas generadas por los profesores fuera de este programa institucional y que aún no han sido registradas.

El propósito del Repositorio de Innovación Educativa es integrar toda esta información para sumar fuerzas y recursos para enriquecer a la comunidad universitaria a través del intercambio de ideas, para romper el aislamiento que es común entre quienes prueban algo "nuevo", así como para lograr generar sinergias que les ayuden a avanzar más rápido.

¿Quieres implementar alguna innovación educativa en tu docencia? ¿Has desarrollado por tu cuenta alguna innovación para la enseñanza o el aprendizaje? Te invitamos a consultar y compartir ideas a través del Repositorio de Innovación Educativa.

\section{Referencias}

* Banerjee B. (2016) Why Innovate? En Banerjee B. \& Ceri S. (eds). Creating Innovation Leaders. Understanding Innovation. Springer International Publishing.

* Barraza, A. (2005). Una conceptualización comprehensiva de la innovación educativa. Innovación educativa, 5(28), 19-31. Recuperado de: http://www.redalyc. org/articulo.oa?id=179421470003.

* Body J., Habbal F. (2016) The Innovation Ecosystem. En Banerjee B. y Ceri S. (Eds), Creating Innovation Leaders. Understanding Innovation (pp. 25-51). Springer International Publishing.

* Casanova Cardiel, H. (2005). La Universidad: Tensiones y futuro. Uni-pluri/versidad, 5(3), 1-9. Recuperado de: https://aprendeenlinea.udea.edu.co/revistas/index. php/unip/article/view/12125.

* Consejo Nacional de Ciencia y Tecnologia (CONACYT). (2020). Repositorio Nacional. Gobierno de México. Recuperado de: https://www.repositorionacionalcti.mx/.

${ }^{5}$ Un entorno inmersivo utiliza la tecnología 3D para reproducir o simular la realidad y permitir que el usuario interactúe con ésta.

- Hernández, M. (2020). Crea la unam la licenciatura en Ingeniería Aeroespacial. Gaceta unAm, 5,116. Dirección General de Comunicación Social. Recuperado de: https://www.gaceta.unam.mx/g20200213/.

* Jisc (s.f.). Opendoar. Directory of Open Access Repositories. Recuperado de: https:// v2.sherpa.ac.uk/opendoar/. 
* Lagzian, F., Abrizah, A., \& Wee, M.-C. (2015). Measuring the gap between perceived importance and actual performance of institutional repositories. Library \& Information Science Research, 37(2), 147-155. Recuperado de: https://doi.org/ https://doi.org/10.1016/j.lisr.2014.06.007.

* Organisation for Economic Co-operation and Development (OECD) (2014). Measuring Innovation in Education: A New Perspective. OECD Publishing. Dol: http:// dx.doi.org/10.1787/9789264215696-en.

* Red Mexicana de Repositorios Institucionales (Remeri) (2012-2020). Red Mexicana de Repositorios Institucionales. CUDI-CONACYT. Recuperado de: http://www.remeri.org. mx/portal/index.html.

- Rogers, E. M (2003). Diffusion of innovations [5a ed.]. Free Press.

* Tierney, W. G., \& Lanford, M. (2016). Conceptualizing Innovation in Higher Education. En M. B. Paulsen (Ed.), Higher Education: Handbook of Theory and Research (pp. 1-40). Springer International Publishing. Dol: doi:10.1007/978-3-31926829-31.

- Universidad Nacional Autónoma de México (unam) (2019). Agenda estadística 2019. Dirección General de Planeación. Recuperado de: https://www.planeacion.unam. mx/Agenda/2019/.

* Zabalza, M. A., y Zabalza Cerdeiriña, M. A. (2012). Innovación y cambio en las instituciones educativas. Homo Sapiens.

\section{Cómo citar este artículo}

- Santos Solórzano, Roberto y González-Flores, Patricia (2020). Innovar en compañía: el Repositorio de Innovación Educativa de la unAm. Revista Digital Universitaria (RDU). Vol. 21, núm. 2 marzo-abril. Dol: http://doi.org/10.22201/codeic.16076079e.2020. V21n2.a9.

Recepción: 17/02/2020. Aprobación: 25/02/2020 